\title{
Research on the thermal insulation of water in bathtub
}

\author{
Hui $\mathrm{Xu}^{1, a}$, Songjing Wang ${ }^{2, b}$ \\ ${ }^{1}$ Faculty of mechanical Engineering \& Mechanics, Ningbo university, Ningbo, 315211, China \\ ${ }^{2}$ Faculty of science, Ningbo University, Ningbo, 315211, China \\ aemail: 459543524@qq.com, bemail: wangsongjing@nbu.edu.cn
}

Keywords: Stochastic Variable, Differential Equation, Statistical Optimization Model, Normal Distribution

\begin{abstract}
In this paper, the bathtub water thermal insulation problem has been studied, it analysis the effect to heat dissipation caused by body movement, and determine the input hot water flow and the output water flow according to the law. Thereby keep the bath water temperature close to the initial one. Firstly, the state that the person keep still in the bathtub is considered. It is equivalent to introducing a boundary condition.
\end{abstract}

With the finite element method, a three dimensional diagram of water temperature distribution under steady state is obtained. Then consider the situation of introducing body movement. The body movement obeys the random distribution, using a random variable to characterize the body movement intensity in the bathtub. Establishing a random differential equation model, using the finite difference method to solve the differential equation. Then a curve about the temperature of the water in a bathtub fluctuate over time is drew. According to the curve, using the statistical optimization model to solve hot water input flow that makes the temperature of the water in a bathtub be as nearly as possible to the initial temperature. Finally, the optimal values of input hot water flow in various initial water temperature is determined.

\section{Introduction}

The bathtub, a common device for holding water, can be regarded as an open-mouth container, which will result in fast heat dissipation due to its big volume and wide water surface. In order to keep the temperature of water in bathtub, two methods are proposed as follows: heat the water in bathtub or input hot water to the bathtub. Reason to maintain the water temperature through heating bath method. The method of heating water in bathtub, due to the wide water surface area, it is not easy to preserve heat. The volume of water in the bathtub is large which consumes a lot of power, and the control process is complex. The manufacturing cost and the use-cost are high, so this method is not feasible. The method of input hot water to the bathtub, and let out the cooling water from bathtub has the advantages of less energy consumption, low performance requirement about material, simple controlling process, and good thermal insulation effect. So this method is practical and feasible.

\section{Natural heat dissipation model of water in bathtub}

Firstly, solve the natural heat dissipation law of water in the bathtub [1],

There are mainly three forms of heat exchange in the bathtub: thermal convection between water and air, heat conduction between water and the bathtub wall, heat boost as hot water input to the bathtub. Thermal convection between water and air can be calculated with the Newton's law of cooling, heat conduction between water and the bathtub wall can be derived from Fourier law of cooling.

Newton's law of cooling is as follows [2]:

$d Q_{1}=q \cdot S_{1} d t=\lambda_{1} S_{1}\left(T-T_{1}\right) d t$ 
Fourier law of cooling is as follows [3]:

$d Q_{2}=\lambda_{2} S_{2} \frac{\left(T-T_{1}\right)}{h} d t$

Uniting formulas (1) and (2), the natural heat loss differential equation of water in bathtub can be obtained as follows:

$\frac{d Q}{d t}=\left(\lambda_{1} S_{1}+\frac{\lambda_{2} S_{2}}{h}\right)\left(T-T_{1}\right)$

In the above expressions, symbol $\mathrm{Q}$ represents the total heat of the water in the bathtub, $Q_{1}$ represents the heat disperses from water to air, $Q_{2}$ represents the heat transfer from water to the bathtub wall, $\lambda_{1}$ is the coefficient of heat dissipation from water to air, $\lambda 2$ is the thermal conductivity between water and the bathtub wall, $S_{1}$ is the contact surface between water and air, $S_{2}$ is the contact surface between water and the bathtub wall, $h$ is the thickness of bathtub wall, $T_{1}$ is the indoor temperature.

\section{Heat dissipation model with heat source adding to the bathtub}

The method of adding hot water to bathtub is adapted to keep the water temperature close to the initial state. The water volume in the bathtub should keep constant, so it is required to drain the cooling water out of the bathtub, and the input water flow is set the same as the output flow. After adding hot water into the bathtub, the distribution of water temperature is not uniform. Firstly, the distribution of water temperature is considered in the case of a person stay still in a bathtub. Water temperature distribution can be determined through the following conditions: convection diffusion equation of water, that is the control equation. The initial state of water in the bathtub. The boundary conditions of the bathtub including heat dissipation between water and air, heat conduction between water and bathtub wall, heat increasing with hot water inputting to the bathtub, and so on. Simultaneously, the water in the bathtub should meet the law of conservation of energy.

Establishing a space coordinate system. And suppose the initial temperature of water at any position in the bathtub is $T_{0}\left(x, y, z, t_{0}\right)$, the water temperature of any time is $T(x, y, z, t)$. When the person keeps still in the bathtub, it is equivalent to introducing a boundary condition to water in the bathtub. Use the notation $T_{p}$ to represent the human body surface temperature. Then three boundary conditions are obtained as follows:

$$
\begin{aligned}
& -\left.k \frac{\partial T(x, y, z, t)}{\partial^{\dagger} n_{1}}\right|_{S_{1}}=H_{1}\left(\left.T(x, y, z, t)\right|_{S_{1}}-T_{1}\right) \\
& -\left.k \frac{\partial T(x, y, x, t)}{\partial^{\dagger} n_{2}}\right|_{S_{2}}=H_{2}\left(\left.T(x, y, z, t)\right|_{S_{2}}-T_{1}\right) \\
& -\left.k \frac{\partial T(x, y, x, t)}{\partial^{\dagger} n_{3}}\right|_{S_{3}}=H_{3}\left(\left.T(x, y, z, t)\right|_{S_{3}}-T_{\mathrm{p}}\right)
\end{aligned}
$$

Formula (4) is a differential equation about heat dissipation between water and air. Formula (5) is a heat conduction differential equations between water and bathtub wall. Formula (5) is the heat transfer equation between humane body and water. $\left.T(x, y, z, t)\right|_{S_{1}}$ represents the temperature of contact surface between water and air, $\left.T(x, y, z, t)\right|_{S_{2}}$ represents the water temperature at the bathtub wall. $\stackrel{v}{n_{1}}$ denotes the normal vector of water surface. $\stackrel{v}{n_{2}}$ represents the normal vector of bathtub wall, $H_{1}$ and $H_{2}$ are proportional constants.

For the convenience of solution, simplifying the person to a cuboid with surface temperature. Because the person is still in the bathtub, it is equivalent to introduce a boundary conditions for the water in the bathtub. Then the person and the bathtub can be seemed as a whole, so part of the boundary of the bathtub is the surface of the body.

With heat water inputting to the bathtub, the convection diffusion equation of water in bathtub is 
as follows:

$\frac{\partial T}{\partial t}+\operatorname{div}(u T)=\operatorname{div}(k \nabla T)$

Temperature of hot water inputting to the bathtub:

$\left.T(x, y, z)\right|_{S_{i}}=T_{i}$

The initial condition of water in the bathtub:

$T\left(x, y, z, t_{0}\right)=T_{0}$

Differential equation of heat dissipation:

$\frac{d Q}{d t}=\left(\lambda_{1} S_{1}+\frac{\lambda_{2} S_{2}}{h}\right)\left(T-T_{1}\right)-C Q_{m}\left(T_{i}-T\right)$

C represents the specific heat of water, $Q_{m}$ represents the hot water flow inputting to the bathtub, $T_{i}$ represents the hot water temperature.

There are some feasible methods can be adopted to solve the above differential equation, such as finite element method [4] finite difference method [5] , characteristic finite difference method [6] characteristic finite element method 、 finite volume method streamline diffusion method[7] and so on. In this paper, the finite element method is adopted to solve the problem. Then draw the three-dimensional diagram about the distribution of water temperature as shown in Fig 1.

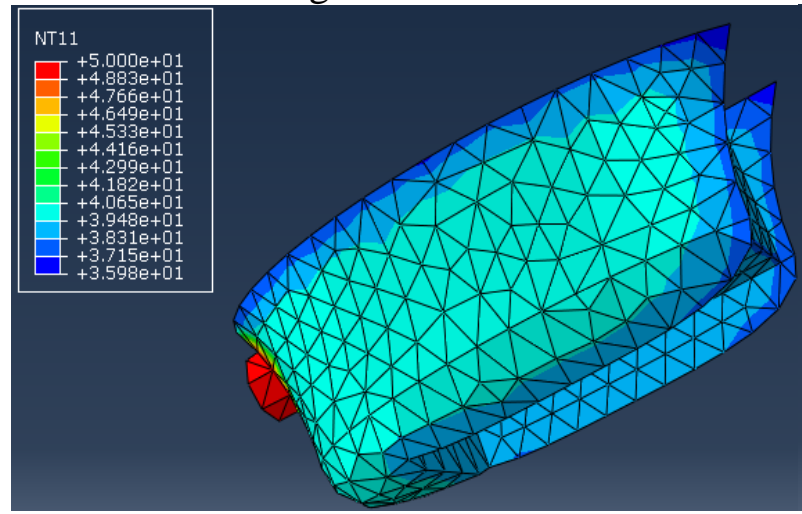

(a)

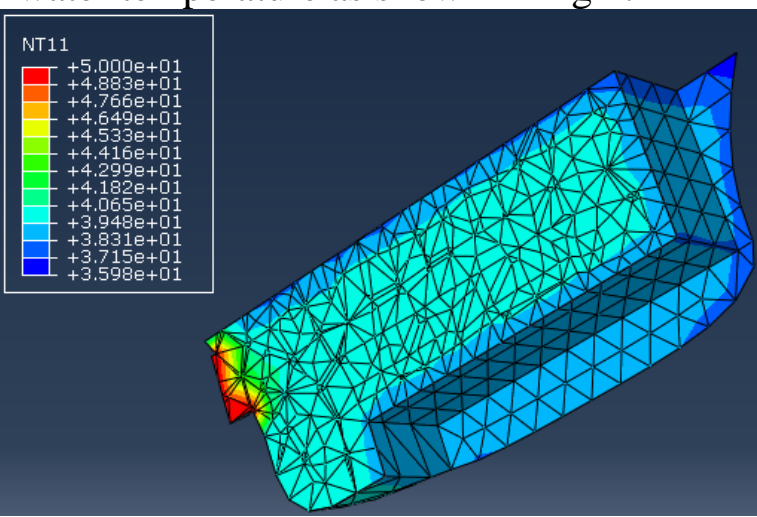

(b)

Fig.1. (a) Three-dimensional diagram of water temperature (b) longitudinal section diagram

Sketch map of temperature distribution in the bath water temperature reaches steady state after the distribution of water temperature in Fig. 1, the concave is the part with the body surface as bathtub boundary. It expresses the boundary conditions for a person keeping still in a bathtub.

\section{Heat dissipation model with interference}

Body movement will accelerate the convection of water in the bath, which speed up uniform distribution of water temperature. So it can be considered that the water temperature in the bath is evenly distributed when the person is moving in the bathtub. Then consider the effect of body movement on heat dissipation. The body movement is random, so set the random motion obey the chi square distribution. The effect produced by the random motion of the person reflected in the heat exchange coefficient. Introducing a constant $\lambda_{C}$ to denote the heat exchange coefficient, which correspond to the water convection caused by body movement. Multiply the coefficient $\lambda_{C}$ by a chi square distribution function $\mathrm{F}(\mathrm{x})$ to describe heat dissipation law with the water being disturbed by random body movement [8]. Then the heat dissipation differential equation with random disturbance is inferred, it is expressed in formula (11).

$$
\frac{d Q}{d t}=\left(\lambda_{1} S_{1}+\frac{\lambda_{2} S_{2}}{h}+F(x) \lambda_{C} S_{1}\right)\left(T-T_{1}\right)-C Q_{m}\left(T_{i}-T\right)
$$

Simulate the model by substituting actual parameters: water volume in the bathtub is 200L, water surface area is $1.7 \mathrm{~m}^{2}$, bathtub wall area is $4.94 \mathrm{~m}^{2}$, the initial temperature of water in the bathtub is 
$40^{\circ} \mathrm{C}$, the indoor temperature is $20^{\circ} \mathrm{C}$, temperature of the hot water inputting to the bathtub is $45^{\circ} \mathrm{C}$, the temperature fluctuations range that people can bear is $\pm 1^{\circ} \mathrm{C}$. take the difference method to solve the differential equation of heat dissipation of the water in bathtub, Then draw the temperature fluctuation curve over time, which is shown in figure 2.

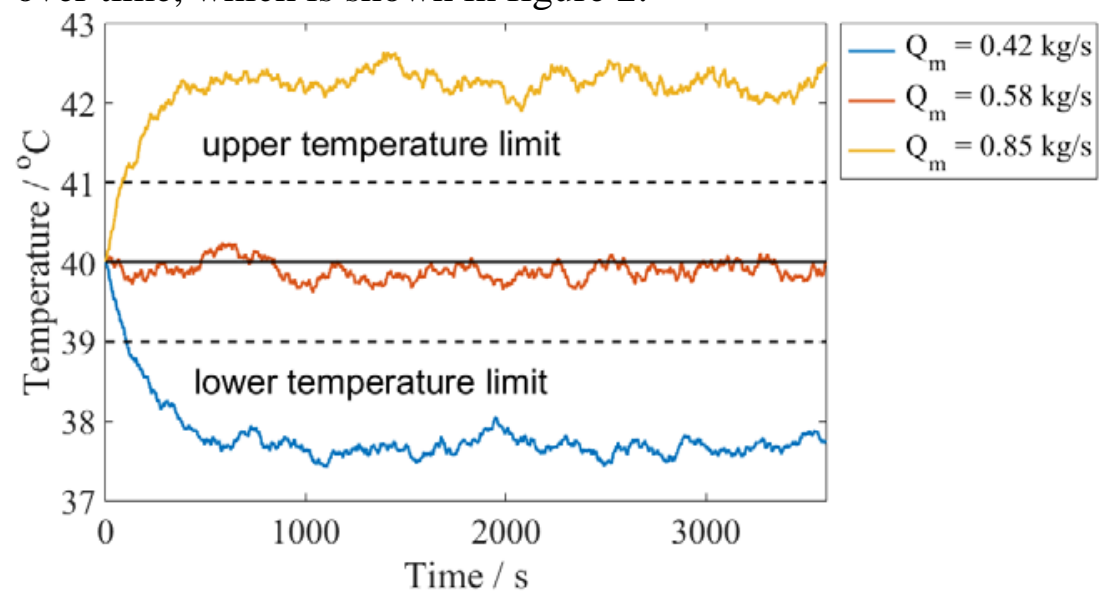

Fig.2. water temperature fluctuates curve over time

The body movement will speed up the heat dissipation, and the hot water added to the bath will increase the energy of the water in the bathtub, so the water temperature will fluctuate, and the fluctuation trend is associated with the intensity of disturbance. Inputting a certain amount of hot water to the bathtub, when the disturbance is vigorous, the water temperature will show a downward trend, When the disturbance is appropriate, the water temperature will fluctuations in the vicinity of the initial temperature, When the disturbance is slight, the water temperature will be showed a rising trend. According to the water temperature fluctuation curve over time, adopting the statistical optimization model [9] to solve the inputting water flow, which can keep the water temperature close to the initial state.

\section{Statistical optimization model to solve the optimal water flow}

Simulate the model with Monte Carlo method. Firstly, setting the flow rate changes from $0.5 \mathrm{~kg} / \mathrm{s}$ to $0.7 \mathrm{~kg} / \mathrm{s}$ at the steps of $0.001 \mathrm{~kg} / \mathrm{s}$, then find the optimal water flow which can keep the temperature in a steady state, and as close as possible to the initial temperature. The state that the water temperature keeps stable can be expressed by the minimum temperature difference between temperature of any time and initial temperature. $T_{0}$ represents the initial temperature of water.

$$
\min \sum\left(T(t)-T_{0}\right)^{2}
$$

Repeat the above process, and perform the experiment 10000 times, and then a distribution map about the experiment times corresponding to different hot water flow that can keep the water temperature steady. From the figure, the optimal hot water flow can be obtained.

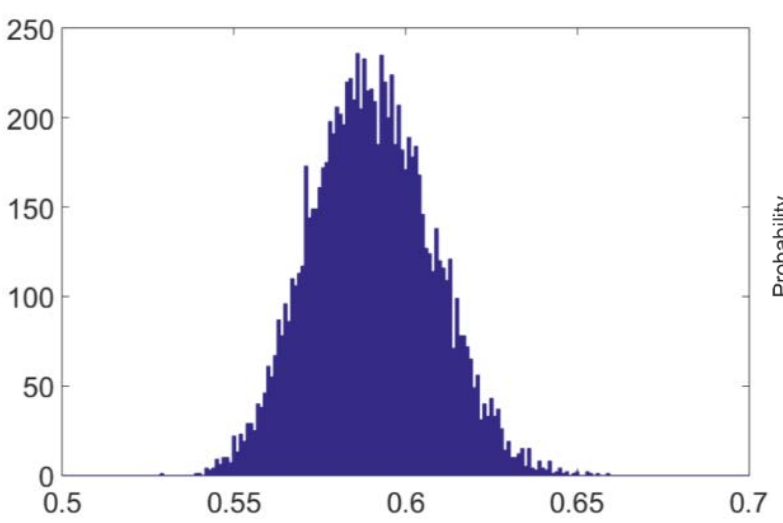

(a)

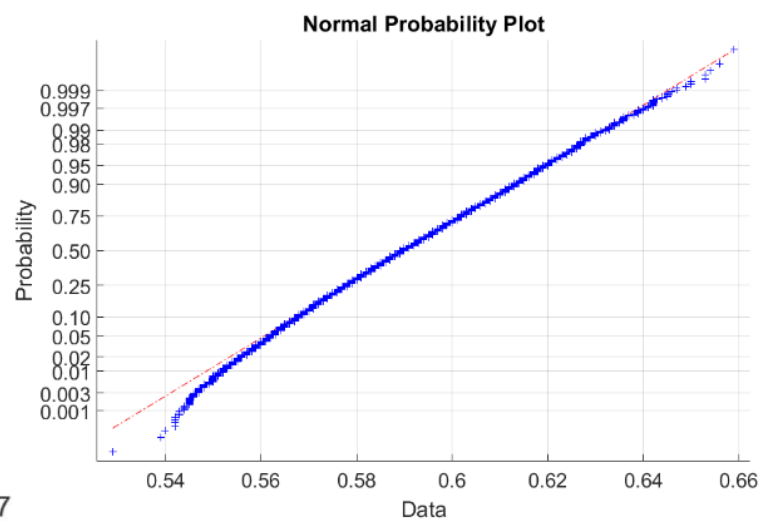

(b)

Fig.3. (a)distribution map about the experiment times corresponding to different hot water flow.

(b)P-P diagram for normal distribution test 
In figure 3(a) the data distribution is approximated by a normal distribution. Taking the P-P diagram [10] to test the distribution of data in Fig3 (a), Fig3 (b) shows the test results, from the diagram it can be seen that all the sample points are approximately distributing near a straight line, so it is concluded that the above data meets the normal distribution.

\section{Result}

The hot water flow inputting to the bathtub is associated with the initial temperature of water and the indoor temperature, it can be expressed with the formula (13).

$Q_{m}=f\left(T_{1}, T_{0}\right)$

Where $Q_{m}$ is the water flow in unit time, here it is used to denote the hot water flow inputting to the bathtub, the unit is $\mathrm{kg} / \mathrm{s}, T_{1}$ the indoor temperature, $T_{0}$ the initial temperature of the water.

Set the hot water temperature $70^{\circ} \mathrm{C}$, substituting the parameters: quantity of a person $50 \mathrm{~kg}$, water volume $200 \mathrm{~L}$, water surface area $1.7 \mathrm{~m}^{2}$, bathtub wall area $4.94 \mathrm{~m}^{2}$ to the above model, then calculate the hot water flow needed on the condition of different initial water temperature and indoor temperature. The initial temperature of water is the goal temperature that should be kept, The result is show in the following table.

Table1. Hot water flow with different initial conditions

\begin{tabular}{rlllll}
\hline T1 & $30{ }^{\circ} \mathrm{C}$ & $35^{\circ} \mathrm{C}$ & $40^{\circ} \mathrm{C}$ & $45^{\circ} \mathrm{C}$ & $50^{\circ} \mathrm{C}$ \\
\hline $10^{\circ} \mathrm{C}$ & $0.09 \sim 0.10$ & $0.17 \sim 0.19$ & $0.28 \sim 0.31$ & $0.47 \sim 0.52$ & $0.82 \sim 0.95$ \\
$15^{\circ} \mathrm{C}$ & $0.09 \sim 0.11$ & $0.18 \sim 0.19$ & $0.28 \sim 0.32$ & $0.47 \sim 0.53$ & $0.83 \sim 0.95$ \\
$20^{\circ} \mathrm{C}$ & $0.10 \sim 0.11$ & $0.18 \sim 0.20$ & $0.29 \sim 0.34$ & $0.48 \sim 0.53$ & $0.82 \sim 0.95$ \\
$25^{\circ} \mathrm{C}$ & $0.10 \sim 0.12$ & $0.19 \sim 0.20$ & $0.29 \sim 0.35$ & $0.48 \sim 0.54$ & $0.82 \sim 0.95$ \\
\hline
\end{tabular}

\section{Conclusion}

Thermal insulation of water in bathtub problem is researched in this paper, Firstly, solve the water temperature distribution on the case of the person keep still in the bathtub, the finite element method is adopted to solve this problem, then the three dimensional diagram about the water temperature distribution in a steady state is obtained. And then establish a differential equation about the heat changes over time with disturbance in the bathtub, and using the difference method to solve this equation, three curves about water temperature fluctuate over time is drew. Then a statistical optimization model is established, with this model, a distribution map about the experiment times corresponding to different hot water flow that can keep the water temperature steady is drew. From the diagram, the optimal value of hot water flow can be determined. In the end, the hot water flow is calculated at different indoor temperature and different initial water temperature, which is convenient for the practical application.

\section{Reference}

[1]. Kroener E, Vallati A, Bittelli M. Numerical simulation of coupled heat, liquid water and water vapor in soils for heat dissipation of underground electrical power cables[J]. Applied Thermal Engineering, 2014, 70(1):510-523.

[2]. O’Sullivan C T. Newton's law of cooling-A critical assessment[J]. American Journal of Physics, 1990, 58(10):956-960.

[3]. Bonetto F, Lebowitz J L, Rey-Bellet L. Fourier's Law: a Challenge for Theorists [J]. 
Encyclopedia of Mathematical Physics, 2000:374-379.

[4]. Ciarlet P G, Oden J T. The Finite Element Method For Elliptic Problems [J]. Mathematics of Computation, 2002, 36(154):460.

[5]. Mjos O D, Faergeman O. Hamilton R L, et al. Visualization of sound pressure distributions in a room by the Finite Difference Method [J]. Journal of Clinical Investigation, 1999, 1999(3):1105-1106.

[6]. Yi-rang Yuan, Chang-feng Li, Cheng-shun Yang, et al. Characteristic finite difference method and application for moving boundary value problem of coupled system [J]. Applied Mathematics \& Mechanics, 2008, 29(5):611-624.

[7]. Zhou G. How accurate is the streamline diffusion finite element method [J]. Mathematics of Computation, 1997, 66(217):31-44.

[8]. [w] Satorra A, Bentler P M. A scaled difference chi-square test statistic for moment structure analysis [J]. Psychometrika, 2001, 66(4):507-514.

[9]. Turkington, B..An Optimization Principle for Deriving Nonequilibrium Statistical Models of Hamiltonian Dynamics [J].Journal of Statistical Physics, 2013. 152(3):569-597.

[10]. Xuping Zong, Yulan Yao. Using Q-Q and P-P diagram to test the statistical distribution of data quickly [J]. Statistics and Decision, 2010, 20:151-152. 